Acta Crystallographica Section D

\section{Biological \\ Crystallography}

ISSN 0907-4449
Igor Nederlof, Eric van Genderen, Yao-Wang Li and Jan Pieter Abrahams*

Biophysical Structural Chemistry, Leiden University, Einsteinweg 55, 2333 CC Leiden, The Netherlands

Correspondence e-mail:

abrahams@chem.leidenuniv.nl

\title{
A Medipix quantum area detector allows rotation electron diffraction data collection from submicrometre three-dimensional protein crystals
}

When protein crystals are submicrometre-sized, X-ray radiation damage precludes conventional diffraction data collection. For crystals that are of the order of $100 \mathrm{~nm}$ in size, at best only single-shot diffraction patterns can be collected and rotation data collection has not been possible, irrespective of the diffraction technique used. Here, it is shown that at a very low electron dose (at most $0.1 \mathrm{e}^{-} \AA^{-2}$ ), a Medipix2 quantum area detector is sufficiently sensitive to allow the collection of a 30-frame rotation series of $200 \mathrm{keV}$ electrondiffraction data from a single $\sim 100 \mathrm{~nm}$ thick protein crystal. A highly parallel $200 \mathrm{keV}$ electron beam $(\lambda=0.025 \AA)$ allowed observation of the curvature of the Ewald sphere at low resolution, indicating a combined mosaic spread/beam divergence of at most $0.4^{\circ}$. This result shows that volumes of crystal with low mosaicity can be pinpointed in electron diffraction. It is also shown that strategies and data-analysis software (MOSFLM and SCALA) from X-ray protein crystallography can be used in principle for analysing electron-diffraction data from three-dimensional nanocrystals of proteins.

\section{Introduction}

Protein crystallography is a major justification for large-scale $\mathrm{X}$-ray facilities such as synchrotrons and free-electron lasers. However, three-dimensional protein crystals that are smaller than about $0.5 \mu \mathrm{m}$ are too small for standard X-ray crystallography, although XFEL sources are expanding the method towards smaller crystals (Chapman et al., 2011). This is a serious bottleneck, as about $30 \%$ of proteins that crystallize do not grow crystals of sufficient size or quality for X-ray structure determination (Rupp, 2004). In particular, membrane proteins and large (dynamic) protein-nucleic acid complexes fail to grow into crystals of sufficient size. Structural information on these important drug targets is therefore severely lacking.

Electrons are less damaging to proteins than X-rays by several orders of magnitude per elastically diffracted quantum (Henderson, 1995). This property of electrons explains the successes of two-dimensional electron crystallography. For instance, $45 \AA$ thick submicrometre patches of twodimensional bacteriorhodopsin crystals yielded images with a resolution of $2.8 \AA$ (Baldwin et al., 1988). Three-dimensional crystals with an equivalent volume would measure approximately $150 \times 150 \times 150 \mathrm{~nm}$. Recent data demonstrate that useful high-resolution electron diffraction data (up to $2.5 \AA$ resolution) can be obtained from nanosized three-dimensional protein crystals, where synchrotron X-rays fail (Jiang et al., 2009).
Received 18 April 2012

Accepted 9 April 2013 


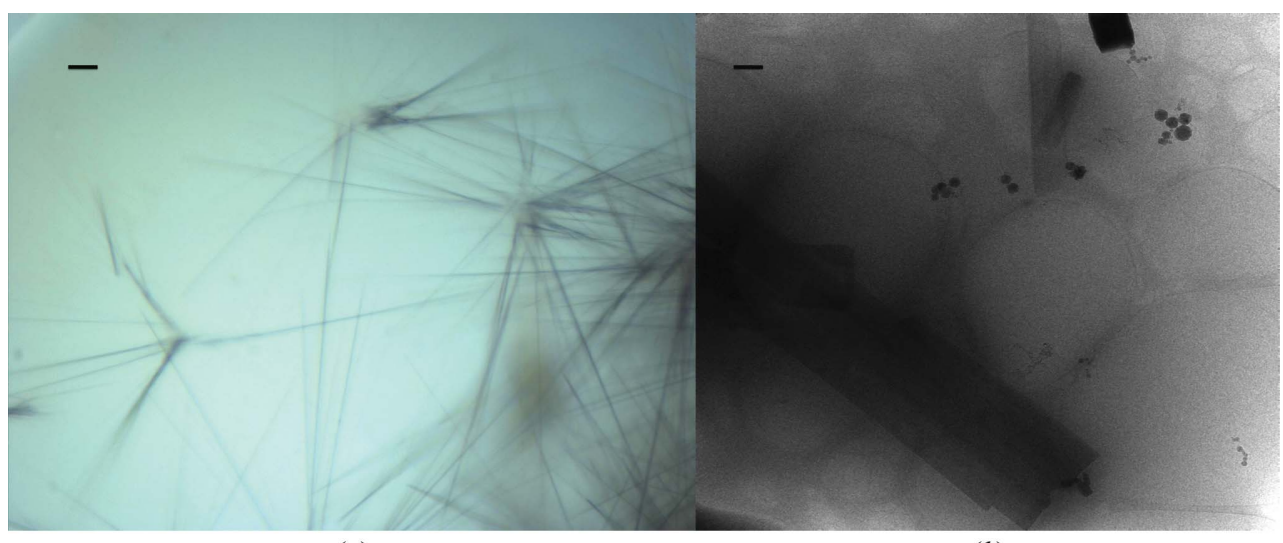

(a)

Figure 1

Lysozyme nanocrystals in a crystallization drop $(a)$ and an electron micrograph of vitrified crystals $(b)$. Scale bars correspond to $10 \mu \mathrm{m}(a)$ and $500 \mathrm{~nm}(b)$.

However, only single diffraction shots could be collected. Collection of rotation data from protein nanocrystals was not possible because the signal-to-noise ratio and dynamic range of CCD detectors and image plates was insufficient. Very recently, direct electron detectors have become available which have a better signal-to-noise ratio and which may be better suited. These new detectors are very expensive and are probably not sufficiently radiation-hard to be routinely exposed to the direct electron beam.

Electrons can also be detected by quantum area detectors such as the Medipix2 (Llopart Cudié et al., 2002; Faruqi \& Henderson, 2007). The Medipix2 detector is more radiationhard than other direct electron detectors such as the Falcon because its read-out electronic circuitry (which is sensitive to radiation damage and interference) is shielded by a semiconductor sensor layer, to which it is bump-bonded (Llopart Cudié et al., 2002). The detector has a very high signal-to-noise ratio because each pixel has its own readout electronics that measures the hole-charges that are produced by an incident electron hitting the sensor layer within $10^{-5} \mathrm{~s}$. If the integrated energy after amplification is above a set threshold corresponding to the energy of a $200 \mathrm{keV}$ electron the incident quantum is counted as a 'hit'. Thus, the Medipix2 chip only counts $200 \mathrm{keV}$ electrons and, unlike many other detectors, is blind to soft X-rays of lower energy that are also produced in great abundance inside any electron microscope. In this fashion its noise is almost exclusively determined by the counting statistics of the electrons. This gives a significant improvement over conventional CCD cameras in electron microscopes (Faruqi \& McMullan, 2011; Georgieva et al., 2011).

We recently showed that a Medipix2 detector with a $500 \mu \mathrm{m}$ sensor layer can detect $200 \mathrm{keV}$ electrons with a signal-tonoise ratio that is at least an order of magnitude better than that of image plate (Georgieva et al., 2011). The Medipix detector is highly sensitive at low count rates, allowing accurate measurement of the high-resolution terms. In addition, with a $500 \mu \mathrm{m}$ sensor layer it is sufficiently radiation-hard to routinely be exposed to a direct beam of $200 \mathrm{keV}$ electrons. It (b)

also has a high dynamic range, allowing accurate measurement of the intense highly peaked dose that it receives in the low-resolution diffraction spots. At $200 \mathrm{keV}$, the point spread of the detector is increased, but if the point spread is not much higher than the spread of the Bragg spots this would not matter for collecting diffraction data.

In view of these desirable characteristics of the Medipix2 detector, we investigated whether it would be possible to reduce the electron dose per diffraction frame by an order of magnitude and still measure high-resolution diffraction data, as this would allow the collection of rotation diffraction data from single protein crystals for the first time.

\section{Materials and methods}

\subsection{Crystallization}

Crystallization experiments were carried out using the sitting-drop vapour-diffusion technique in Innovadyne SD-2 plates. The Rock Maker software (Formulatrix) was used to design the experiments. A Genesis (Tecan) robot was used to dispense the screening solutions in the reservoirs of MRC2 plates (Swissci). An Oryx 6 (Douglas Instruments) crystallization robot was used to transfer $500 \mathrm{nl}$ reservoir solution and $500 \mathrm{nl}$ protein solution into sitting-drop wells. Plates were stored at $291 \mathrm{~K}$ and imaged using a Rock Imager automated imaging system (Formulatrix). Lysozyme $\left(8 \mathrm{mg} \mathrm{ml}^{-1}\right)$ formed needle-shaped crystals after $48 \mathrm{~h}$ when mixed in a $1: 1$ ratio with well solution consisting of $0.1 \mathrm{M}$ sodium acetate $\mathrm{pH} 3.8$, 1.0 $M$ potassium nitrate (Fig. 1).

\subsection{Vitrification}

Protein crystals were vitrified using a Vitrobot (FEI). $3 \mu \mathrm{l}$ well solution was mixed with the drop containing nanocrystals and transferred to a $3 \mathrm{~mm}$ glow-discharged holey carbon grid (AGAR). Excess liquid was blotted away (blot time $3 \mathrm{~s}$, blot force 5) and the sample was plunge-frozen in liquid ethane cooled by liquid nitrogen.

\subsection{Rotation electron diffraction}

Diffraction data were collected at $200 \mathrm{keV}$ on a CM200FEG (Philips) transmission electron microscope at the National Center for High Resolution Electron Microscopy in Delft. Samples were cooled to $93 \mathrm{~K}$ in liquid nitrogen in an in-housemodified cryo-transfer holder (Gatan). Diffraction patterns were collected using a Medipix2 detector. We created a narrow highly parallel electron beam with limited intensity by using a small $(10 \mu \mathrm{m})$ condenser aperture and spot size 11 (which controls the first condensor lens). The beam was hardly 
convergent, as indicated by the data analysis described below $\left(<0.4^{\circ}\right)$. An automated compu-stage allowed crystal alignment and rotation.

\subsection{Measuring electrons using a Medipix2 detector}

Diffraction patterns were collected with a CMOS Medipix2 detector mounted on a Nikhef carrier board (Fig. 2) and the data were transferred to a Windows PC using USB1.1 read-out electronics (Vykydal et al., 2006). Four abutting Medipix2 ASICs, each with $256 \times 256$ pixels and a pixel size of $55 \mu \mathrm{m}$, make a Medipix2 Quad. The Quad was covered by a single custom-made $500 \mu \mathrm{m}$ semiconductor sensor chip. The distance between two neighbouring single Medipix2 ASICs is approximately $250 \mu \mathrm{m}$, so the edge pixels are about $125 \mu \mathrm{m}$ wide. Therefore, they will capture more signal than the other pixels, resulting in a bright cross that quarters the raw images. This cross can be corrected for, as discussed below.

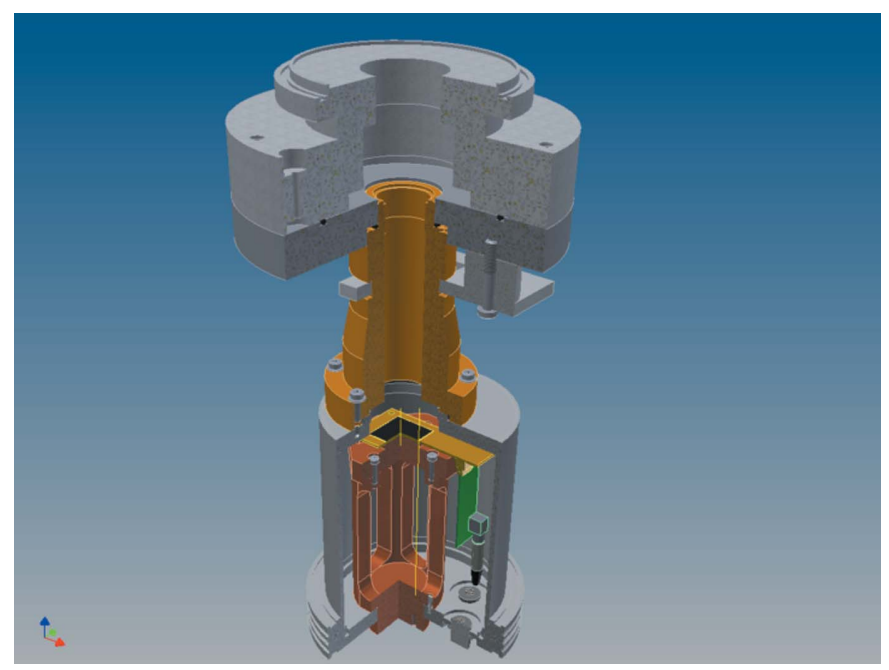

Figure 2

Cutout view of the Medipix2 camera assembly. Electrons enter the tube from the top and hit the detector (black square) on the Nikhef assembly board (San Segundo Bello et al., 2003), which is connected to the computer through the USB read-out electronics and a vacuum connector.
The detector has a large dynamic range, where each pixel consists of a separate 14-bit pseudo-random counter. The test circuitry and four-bit trimming system are able to compensate for most fabrication variations and therefore the overall global threshold has a variation of $95 \mathrm{eV}$. The electronics noise can account for another $110 \mathrm{eV}$. These two values combined result in a dynamic range of no lower then $900 \mathrm{eV}$ between channels. In silicon $\sim 3.6 \mathrm{eV}$ is required to produce one electron-hole pair; therefore, a $200 \mathrm{keV}$ electron can produce $\sim 55000$ pairs. This means that the dynamic range of the electronic noise accounts for less than $1.6 \%$ of the total deposited energy per electron incident and even less when there is more than one electron incident per clock cycle (McMullan et al., 2007; Plackett et al., 2010).

The thickness of the sensor layer $(500 \mu \mathrm{m})$ is larger than for stock Medipix2 chips $(300 \mu \mathrm{m})$. We selected this larger thickness to prevent the $200 \mathrm{keV}$ electrons from penetrating through the sensor layer and damaging the underlying electronics. Monte Carlo simulations ( $300 \mu \mathrm{m}$ Si sensor layer; 120 , 200 and $300 \mathrm{keV}$ ) predicting such events have been described previously (Faruqi \& Henderson, 2007; McMullan et al., 2009; Turecek et al., 2011). Most importantly, they also describe events where these higher energy electrons scatter in the silicon layer to neighbouring pixels. As long as this scattering stays close to the boundaries of the Bragg spots, this would give no major point-spread problems.

There is a need to set the threshold settings such that the gain of the detector is close to or slightly lower than one. Setting the threshold too low will result in multiple pixels recording a single high-energy electron hit; setting the threshold too high will not count electrons or (at suboptimally high thresholds) will only count electrons that deposit all of their energy into a single pixel. It is important that the threshold is chosen in such a way that when the electron partly scatters to a neighbouring pixel it still is counted. The chance of a single high-energy electron scattering in the sensor layer to neighbouring pixels increases with higher energies (Faruqi \& McMullan, 2011). To obtain a higher electron-counting gain, a compromise threshold level

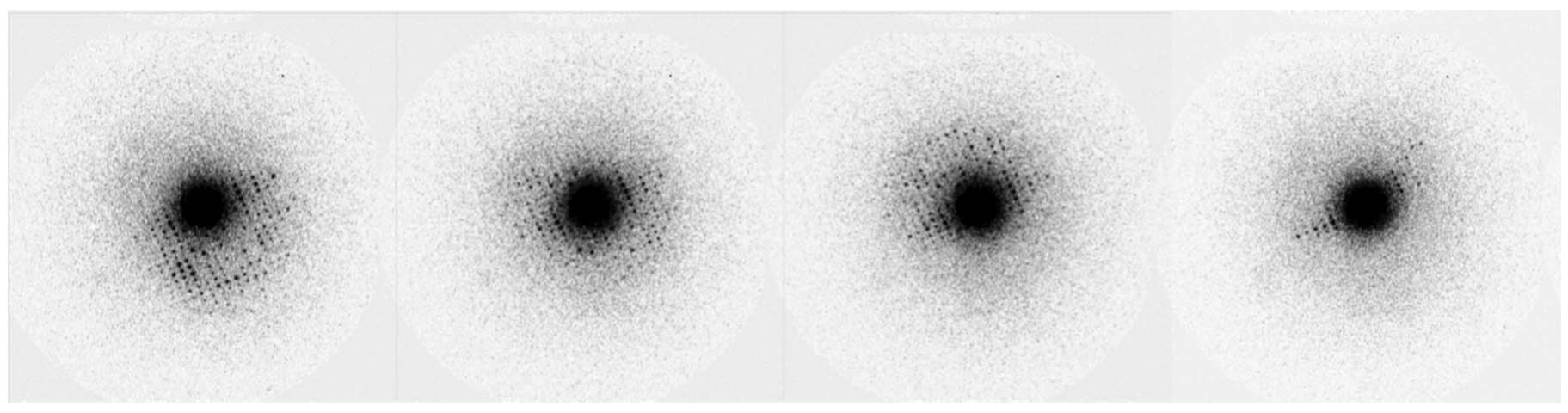

Figure 3

Four diffraction patterns from a series of 23 consecutiveframes $\left(0.05^{\circ}\right.$ per frame, $0.083^{\circ}$ between adjacent images; see text for details $)$ collected at $200 \mathrm{keV}$ with a Medipix 2 Quad detector $(512 \times 512$ pixels). The crystal was orthorhombic lysozyme, maximum resolution $\sim 1.8 \AA$. The frame on the far right is after $30 \mathrm{~s}$ of data collection and shows the degradation in signal arising from beam damage. Each frame was exposed to between 0.05 and $0.1 \mathrm{e}^{-} \AA^{2}$ per second. 
was chosen where lower energy particles would also be counted in each pixel. This, however, is not of much influence in electron-diffraction studies with the Medipix, since most other particles (X-rays) will stay well below an equivalent $100 \mathrm{keV}$ threshold setting. This would be an undesirable setting in any case, because it would enable double counting in neighbouring pixels for a single-electron hit. To minimize radiation damage the experiment was conducted under such low-dose conditions that the chance of a two-electron hit within the same pixel within a clock cycle of the camera is almost non-existent (e.g. less then $0.1 \%$ ). We validated our gain estimate with MOSFLM. The spot-profile statistics predicted a gain of 0.816 counts per electron. However, since the measurement was performed at very low dose conditions (the average count per pixel per frame is around five) it is no longer possible to estimate the gain by fitting a Gaussian (the MOSFLM method) instead of a Poisson distribution. Therefore, the true gain must be higher than 0.816 but not higher than 1.00 .

The vacuum pod in which the Medipix2 was mounted on the on-axis port of the CM-200 FEG electron microscope is shown in Fig. 3. It houses both the carrier and the USB1.1 readout board. Damage to the electronics of these boards by the direct electron beam was prevented by the small window of the pod, which only allowed illumination of about $80 \%$ of the total area of the Medipix2. Out-gassing of the electronics at $1 \times 10^{-6} \mathrm{~Pa}$ vacuum did not prove to be a problem for either the electronics or the TEM. The bias voltage on the Medipix2 was set to $100 \mathrm{~V}$ to be able to count the holes after the electron incidents (Georgieva et al., 2011). The four chips are set to nonparallel readout by the Pixelman v.2.0 software (Turecek $e t$ al., 2011 ) on a PC with Windows 7 in Java mode. The Medipix 2 Quad was calibrated using the standard tools that are included in Pixelman.

(i) DACs were scanned to determine the noise edge of the different ASICs.

(ii) Threshold equalization was performed to remove small standard discrepancies between individual pixels ( 3 bits). This procedure adjusts each pixel so that its threshold is as close as possible to the average and was performed with the standard settings supplied with the Pixelman package.

(iii) Each lower threshold (THL) was set to a value close to the noise edge in order to acquire as much signal as possible from the incident electrons.

(iv) Flat-field images obtained by illumination with a uniform beam allowed equalization of the four chips.

\subsection{Preparing diffraction patterns for data processing}

The Medipix 2 images need some pre-processing before they can be read into MOSFLM. Three problems needed to be addressed: (i) the cross needed to be removed, (ii) dead pixels needed to be corrected and (iii) the image needed to be centred, as there was a substantial drift of the direct beam. With this aim, we wrote a program that solved these issues as described below.
2.5.1. Removing the cross. A bright cross appears in the raw Medipix2 Quad images as it is a tiled assembly of four single Medipix2 ASICs. The edge pixels of each single Medipix2 ASIC that abuts another in the Quad assembly are larger than the other pixels (they are $125 \mu \mathrm{m}$ wide rather than $55 \mu \mathrm{m}$ ). Therefore, the pixels at the interface between single Medipix2 ASICs capture more electrons. This results in spatial distortion and non-uniformity of the measured signal. In order to correct for the spatial distortion, our program shifts pixels with an $x$ value smaller than 256 by one pixel to the left and shifts pixels with a value of 256 or higher by one pixel to the right. It applies vertical shifts in a similar fashion. This procedure duplicates the pixels adjacent to the horizontal and vertical bisecting lines (and quadruples the four centre pixels). Their value was divided by 2.3 (or 5.3 for the quadrupled centre pixels) to correct the increased measured intensities of these pixels.

2.5.2. Correcting bad pixels. Despite tuning the pixels of the Medipix2 detector, it can still have dead pixels (or bright pixels). Since the positions of dead pixels are fixed and their values do not follow a Poisson distribution over time, it is possible to identify these dead pixels. For each pixel position, we calculated its variance over a range of images and compared this variance with the variances at the other positions. 'Good' pixels will have a much higher variance than 'bad' pixels since the values of the latter tend to be relatively constant and largely independent of the signal, although an occasional bad pixel may be hypervariable with a large variance. If the variance value was below a set threshold the pixel was labelled as 'bad'. After having identified the bad pixels, every image was corrected. For every bad pixel in every image, its value will be replaced by the mean value of the good pixels that surround it. If a bad pixel had no good neighbours the procedure was iterated after reclassification of the pixels.

2.5.3. Centring the images. The position of the beam centre shifted as a function of the tilt of the sample holder. The shift was not huge (15 pixels maximally), but was sufficiently large to interfere with data processing. Images were centred by calculating their cross-correlations with a two-dimensional Gaussian function peaking at pixel $(256,256)$ and applying the calculated shift. After centring the images, they were written to disk in the CCP4 .pck format (Abrahams, 1993) as $1200 \times$ 1200 pixel images so that they could be processed as small MAR images by MOSFLM (Leslie \& Powell, 2007).

\section{Results}

\subsection{Collecting diffraction data}

Prior to installing the Medipix2 detector in the electron microscope, we collected diffraction data on an image plate, film and CCD, but with these detectors we were never able to collect multiple diffraction patterns from single protein crystals to high resolution because of beam damage (Jiang et al., 2009). The Medipix 2 detector has an efficiency at low count rates that is at least an order of magnitude higher than that of an image plate (Faruqi \& McMullan, 2011; Georgieva et al., 
2011). This meant that for the first time we could collect rotation data over multiple images without severe beam damage.

Unfortunately, the hardware prevented us from interfacing the detector with the electron illumination and the rotation of the sample holder. This meant in practice that we had to start rotating the crystal slowly, switch on the beam and start collecting images until the crystal had sustained too much damage. Because transferring a single Medipix 2 frame into the computer takes $0.7 \mathrm{~s}$ (we were using a USB1 interface), this meant that we could only collect rotation data with angular gaps between adjacent images. By using fine- $\varphi$ slicing, we reduced the systematic errors that were introduced by this unfortunate but in the circumstances inevitable experimental flaw.

We collected rotation data from many crystals, improving the data-collection strategies and sample handling iteratively. Here, we describe representative results using two rotation data sets from the same single crystal but collected at different

positions using a fresh part of the crystal. We rotated in the positive direction for the first wedge of data and in the negative direction for the second wedge. Both wedges started at the same goniometer setting, but processing revealed their orientations to be about $1.5^{\circ}$ apart. The first wedge was measured with a rotation speed of $0.083^{\circ}$ per image $\left(0.05^{\circ} \mathrm{s}^{-1}\right)$, reading out an image every second. This resulted in a data set in which each image had recorded about $0.050^{\circ}$ of rotation data, with a gap of $0.033^{\circ}$ until the next image. For the second data set the crystal was rotated at a speed of $0.2^{\circ} \mathrm{s}^{-1}$, reading out an image every second. This resulted in a data set with $0.15^{\circ}$ per image and gaps between images of $0.11^{\circ}$. Both data sets had diffraction spots to a resolution of $1.8 \AA$ in the early images (see Fig. 4).

The diffraction spots were analysed and the profiles of two single spots are shown in Fig. 5. Low-intensity spots (15-20 electrons in the peak) that are far from the central beam clearly show up above the noise (Fig. $5 a$ ). These spots still show a significant level over the surrounding background pixels, which on average count

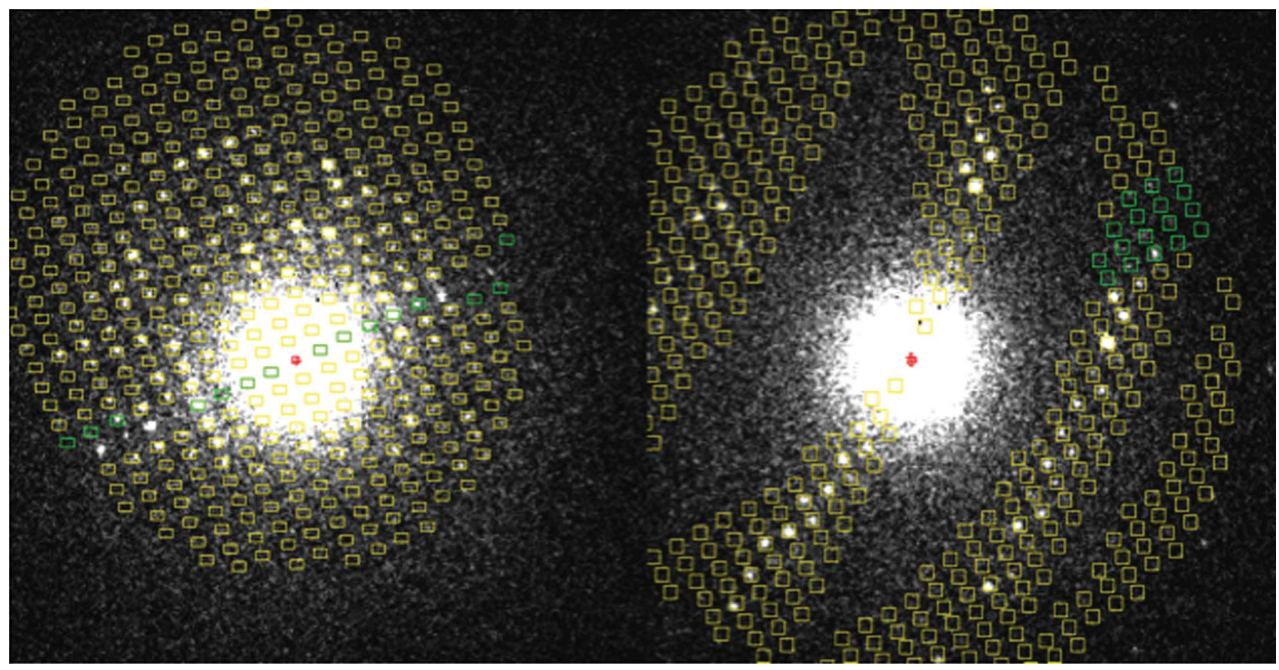

Figure 4

Overlay of predicted spot positions (resolution threshold $2 \AA$ ) with observed diffraction patterns of lysozyme nanocrystals, indicating that the $a$ axis could be close to $125 \AA$, while the $b$ and $c$ axes are about 34.2 and $25.5 \AA$, respectively. The images are screen shots of MOSFLM runs.
2-3 electrons. For comparison, a bright spot with a maximum intensity of 100-120 electrons per pixel is shown in Fig. 5(b).

\subsection{Processing the diffraction data with MOSFLM}

Several essential parameters had to be extracted from the diffraction patterns: the angle of the rotation axis relative to the detector coordinate frame, the beam centre, the beam divergence, the unit-cell parameters and the orientation of the crystal. The determination of each of these parameters will be discussed in more detail below.

3.2.1. Beam centre. We observed the beam centre to shift

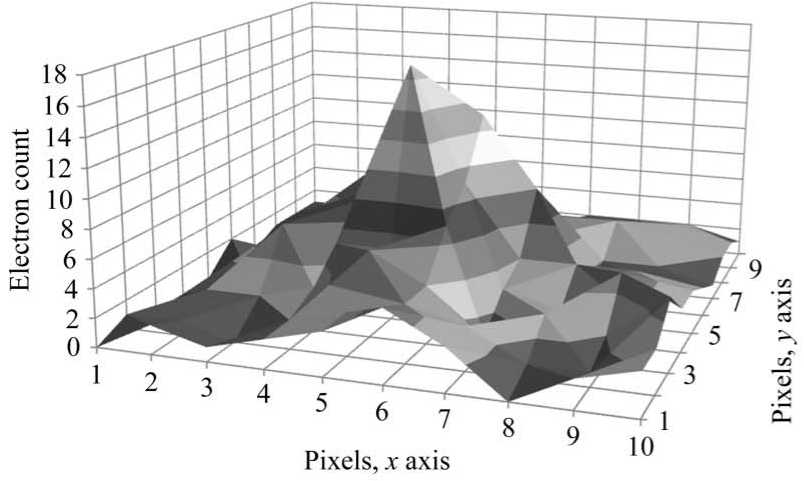

: $0-2=2-4=4-6=6-8=8-10=10-12=12-14=14-16=16-18$

(a)

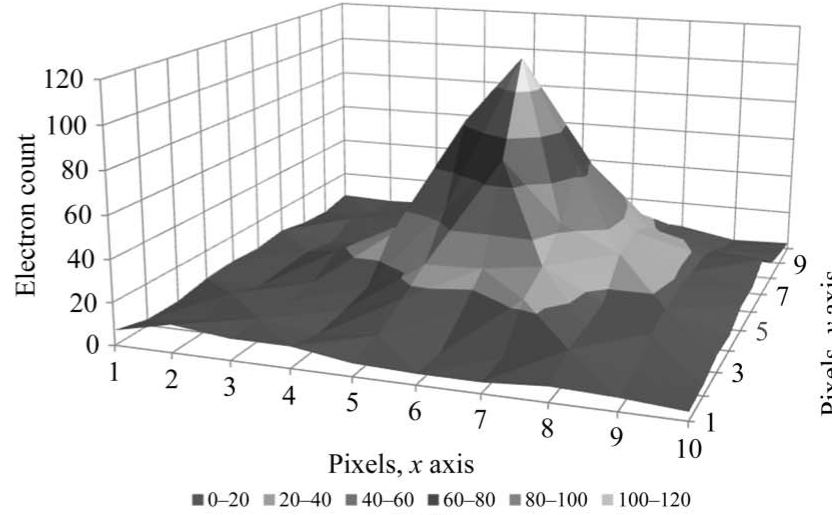

(b)

Figure 5

Two typical spot profiles in a $10 \times 10$ pixel frame. (a) A low-intensity spot profile at 2.0 A resolution with a maximum of $15-20$ electrons. (b) A highintensity spot profile with a maximum of $100-120$ electrons close to the central beam. 
considerably (about 15 pixels) between the starting and ending rotation angles. Since we did not use a backstop, this shift could readily be corrected after data collection by shifting back the images accordingly (as described in \$2). At the time of data collection it was unclear what the cause of the beam shift was, but it later transpired that a screw of the EM stage close to the crystals had become slightly magnetized.

3.2.2. Angle of the rotation axis. As the electrons spiral their way through the magnetic lenses of the microscope, the rotation axis that is observed on the detector is not necessarily an orthogonal projection of the physical rotation axis. It needs to be calibrated for every camera distance. Careful analysis of

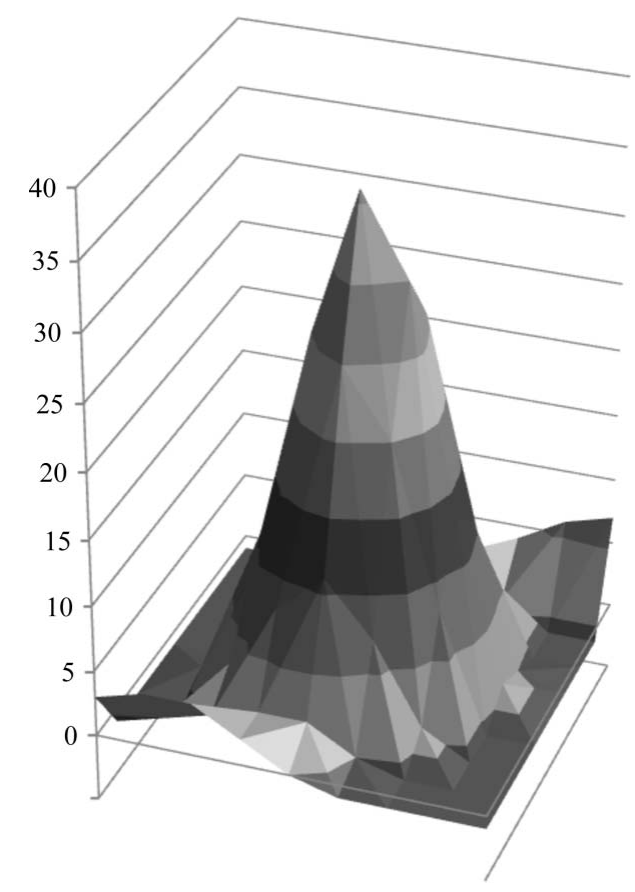

Figure 6

Spot profile from MOSFLM for the first data set. The second data set had a similar spot profile (not shown).
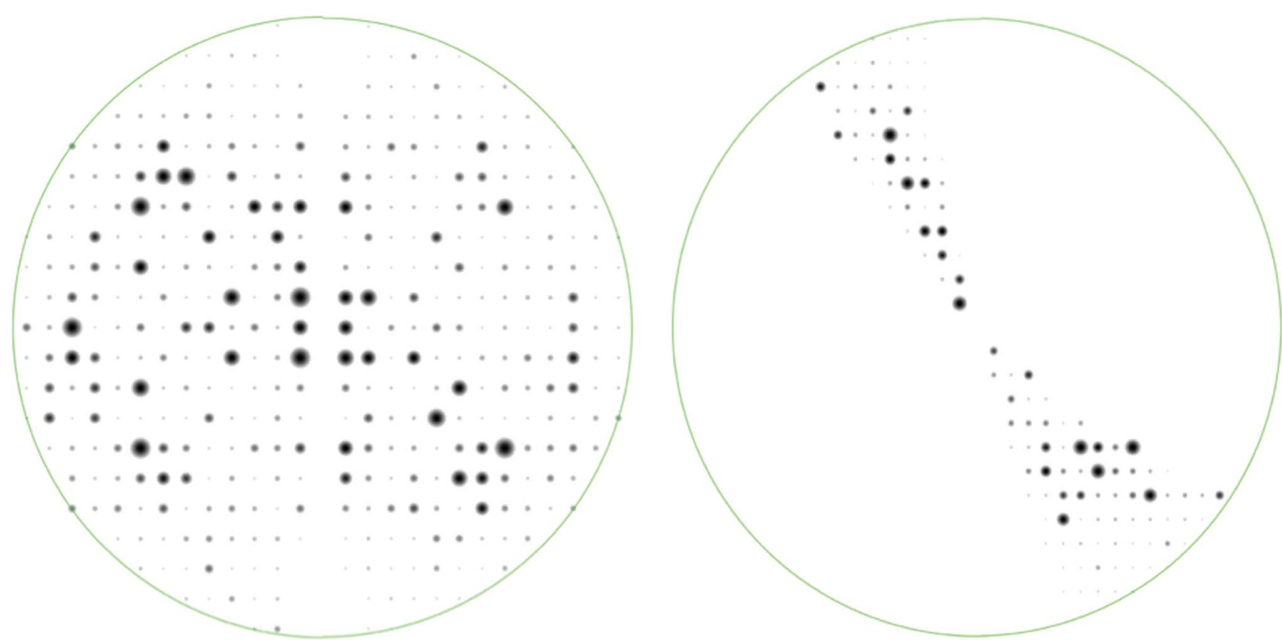

Figure 7

Laue zones at $h=0$ of the scaled data as displayed by VIEWHKL. Note that the second data set (right pattern) does not have a complete $h=0$ Laue zone. the diffraction patterns using the human eye, a ruler and a protractor indicated that at a virtual camera length of $700 \mathrm{~mm}$ and an electron energy of $200 \mathrm{kV}$ the angle between the rotation axis and the $x$ direction of the detector was around $115^{\circ}$. We could refine this initial estimate to $116.5^{\circ}$ by overlaying predicted diffraction patterns on observed diffraction patterns once we had good estimates of the other parameters. When rotating in the negative direction, the rotation axis was redefined to be $-63.5^{\circ}$.

3.2.3. Unit-cell parameters. Using our knowledge of the virtual crystal-to-film distance and the wavelength (which is $0.0251 \AA$ for electrons at $200 \mathrm{keV}$ energy), we interactively estimated the two spacings in the plane of the detector using the 'measure' facility in MOSFLM. These parameters (34.2 and $25.5 \AA$ with an angle of $90^{\circ}$ ) were consistently found in both rotation ranges. No low-index spacings of the third axis were present in the images, and its magnitude could only be estimated from the location of the lunes in the second data set. A reasonable, but by no means perfect, overlay between observed and predicted spot positions could be obtained with a unit cell of $a=125, b=34.2, c=25.5 \AA$, with all cell angles $90^{\circ}$ (Fig. 4). In the past we found orthorhombic nanocrystals of lysozyme to have $P 2_{1} 2_{1} 2_{1}$ symmetry, with a unit cell of around $a=31.5, b=52.5, c=89 \AA$ (Jiang et al., 2009). However, we could not index the rotation data with the latter unit-cell parameters.

3.2.4. Determining the orientation of the crystal. Owing to radiation damage, we could only measure small wedges of data. Auto-indexing routines could not cope with the data. Therefore, we indexed by eye and let MOSFLM refine the orientation using post-refinement, which, despite the angular gaps between images, managed to improve the fit between the observed and predicted patterns. The wedges contained sufficient data for orientation refinement, but did not allow refinement of the unit-cell parameters.

3.2.5. Beam divergence. Once we had satisfactory predictions of the diffraction patterns, we adjusted the beam divergence (or mosaic spread; the two phenomena cannot easily be disentangled) in MOSFLM so that all spots were covered by the predicted pattern. The combined beam divergence/mosaic spread was about $0.3-0.4^{\circ}$.

Typical profiles of the spots are shown in Fig. 6. There were a number of bad spots, mainly because of a high gradient in the background, but by setting the maximum gradient to five (instead of the default value of three) all spots were accepted. One likely cause of the high background gradient for some of the spots was the very intense zero-order reflection. Removing this reflection with a backstop 
was not possible without also removing all of the reflections with a resolution lower than about $5 \AA$. The reason for this is the low scattering angle and the position of the backstop in the CM-200 microscope. For protein crystallographic data collection the position of the backstop should have been much closer to the detector, but hardware restrictions prevented us from moving the backstop.

Scaling the data was problematic in view of the high partiality, the small wedge size, the scarcity of data and the angular gaps between the frames. For this purpose, we used the CCP4 program SCALA (Evans, 2006). The statistics were poor because we had to scale the partials using their calculated partiality and because of the large missing gaps of data between adjacent images. Fig. 7 shows the merged and scaled $h=0$ zones of both data sets. Note that we processed all of the data as $P 1$ with anomalous signal. We did so in order to identify potential dynamic scattering, which results in a breakdown of point-group symmetry in the diffraction patterns (Abrahams, 2010). The symmetries shown in Fig. 7 are therefore intrinsic to the diffraction experiment and not imposed by symmetry averaging of corresponding reflections.

\section{Discussion and conclusion}

Three-dimensional nanocrystals of lysozyme have successfully been grown, vitrified and transferred to an electron microscope at liquid-nitrogen temperature. Rotation electrondiffraction data were obtained at multiple positions of a single submicrometre crystal to resolutions better than $2 \AA$. For the first time, we could collect multiple frames from protein nanocrystals. This was made possible by the much higher sensitivity and signal-to-noise ratio of the Medipix2 detector, combined with its radiation hardness at $200 \mathrm{keV}$.

By using a parallel electron beam, we could observe the curvature of the Ewald sphere in electron-diffraction patterns of protein crystals for the first time. At $200 \mathrm{keV}$, electrons have a wavelength only about $0.025 \AA$, causing the Ewald sphere to be extremely flat at low resolution. Yet the data show that the curvature is still significant.

When the curvature of the Ewald sphere cannot be ignored, both members of a Friedel pair cannot simultaneously be in full diffraction. Geometric considerations imply that the rotation angle between the two reflections of a Friedel pair is always at least their diffraction angle. Consider, for instance, a reflection at $5 \AA$ resolution and assume that it is optimally aligned. At $\lambda=0.025 \AA$ the crystal would need to be rotated by almost $\sin ^{-1}(0.025 / 10)=\sim 0.3^{\circ}$ in order to move its Friedel mate into full diffraction. The fact that we can clearly see deviations in intensity between Friedel mates even at $5 \AA$ resolution (Fig. 4) confirms the low beam divergence and mosaic spread suggested by the data processing. Clearly, the rocking curve of the crystal (with a maximal width of less than $0.4^{\circ}$ ) is sufficiently peaked to allow the observation of these fine angular differences in orientation. This implies that it should be possible to pinpoint areas of low mosaicity in the crystals. This may have an advantage if the crystalline order varies within a single nanocrystal, in which case its best parts can be selected.

These results are encouraging enough to consider fixing the technical problems that prevent optimal data collection. The lack of a scheduling interface between the detector and the goniometer that controls the crystal orientation, combined with the relatively slow readout of the Medipix2 Quad ( $0.7 \mathrm{~s})$, is an evident cause of many of these problems. As the rotation of the sample could not be interrupted during the readout time, there were angular gaps between subsequent frames. We anticipate fixing this problem by employing a novel Medipix 2 detector on a Relaxd read-out board (Visser et al., 2011) with improved readout time (up to 200 frames per second) that can also read data while collecting the next frame. This would significantly decrease the illumination time and subsequent beam damage. We plan to mount the detector on a Titan Krios machine, so data collection will be improved owing to the presence of an autoloader, a programmable goniometer and a programmable beam blanker. For small-molecule crystals, it has been reported that the collection of data in STEM diffraction mode further reduces beam damage (Kolb et al., 2011), so we plan to incorporate the Medipix2 detector in a microscope that has this option.

In view of the suboptimal data collection, we were happy being able to integrate the data with one of the standard programs for processing X-ray data: MOSFLM. However, we cannot exclude the possibility that the results that we report here do not reflect the true crystal structure. We noticed unexpected correlations between parameters. For instance, rotation of the crystal around $\varphi$ was accompanied by an apparent rotation (as suggested by post-refinement) around $\Phi X$, which is a rotation of the crystal around the beam. This may perhaps be caused by eucentric height variation of the crystal as it is being rotated. Since the electrons are focused spirally, rotation of the image or diffraction pattern with height is expected. These and other correlations, which were independent of the unit-cell or crystal-orientation parameters, may indicate that the indexing solution that we present here is wrong. However, we think that these correlations are caused at least in part by distortions specific to electron diffraction. These exist and we have unequivocally identified at least one. For instance, the correlation of the beam shift with rotation of the crystal, which we have observed (and corrected for), would never be encountered in X-ray diffraction.

Together with beam damage, the angular gaps between adjacent images may explain the observed breakdown in point symmetry observed in the $h=0$ Laue zones (Fig. 7). An alternative explanation may be found in the effects of dynamic scattering, which also causes such a breakdown in symmetry (Abrahams, 2010). With improved hardware we anticipate being able to distinguish between these effects.

We have demonstrated that it is possible, at least in principle, to collect rotation electron-diffraction data from protein nanocrystals. This prompts the question concerning the usefulness of such data sets in solving protein crystal structures. Problems with dynamic scattering have been anticipated, yet these did not prevent structure solution of the 
aquareovirus to $3.3 \AA$ resolution by single-particle analysis (Zhang et al., 2010) using crystals that also had a size of approximately $100 \mathrm{~nm}$, just like those analyzed here. If at higher resolution the effects of dynamical scattering can no longer be ignored, they can be modelled by the multi-slice approach (Jansen et al., 1998). This is common practice in electron crystallography of small molecules. Furthermore, there are theoretical considerations that imply that dynamical scattering may yield phase information (Abrahams, 2010) and a limited amount of such dynamical scatter may therefore even be beneficial.

Electron area detectors such as Medipix that allow the distinction of the signal of high-energy electrons from that of other types of radiation are likely to have a major impact on structural biology. Our results imply that protein crystallography also stands to benefit from these technical advances.

This research was funded in part by an NWO-TOP grant (TOP.08.B3.014) and an STW grant (STW 07326). We gratefully acknowledge the help of Professor Zandbergen, Dr Ir Tichelaar and Mr De Kruijff for kind assistance at the National Center for High Resolution Microscopy in Delft.

\section{References}

Abrahams, J. P. (1993). Jnt CCP4/ESF-EACBM Newsl. Protein Crystallogr. 28, 3-4.

Abrahams, J. P. (2010). Z. Kristallogr. 225, 67-76.

Baldwin, J. M., Henderson, R., Beckman, E. \& Zemlin, F. (1988). J. Mol. Biol. 202, 585-591.

Chapman, H. N. et al. (2011). Nature (London), 470, 73-77.

Evans, P. (2006). Acta Cryst. D62, 72-82.

Faruqi, A. R. \& Henderson, R. (2007). Curr. Opin. Struct. Biol. 17, 549-555.

Faruqi, A. R. \& McMullan, G. (2011). Q. Rev. Biophys. 44, 357-390.
Georgieva, D., Jansen, J., Sikharulidze, I., Jiang, L., Zandbergen, H. W. \& Abrahams, J. P. (2011). J. Instrum. 6, C01033.

Henderson, R. (1995). Q. Rev. Biophys. 28, 171-193.

Jansen, J., Tang, D., Zandbergen, H. W. \& Schenk, H. (1998). Acta Cryst. A54, 91-101.

Jiang, L., Georgieva, D., Zandbergen, H. W. \& Abrahams, J. P. (2009). Acta Cryst. D65, 625-632.

Kolb, U., Mugnaioli, E. \& Gorelik, T. E. (2011). Cryst. Res. Technol. 46, 542-554.

Leslie, A. G. W. \& Powell, H. R. (2007). Evolving Methods for Macromolecular Crystallography, edited by R. J. Read \& J. L. Sussman, pp. 41-51. Dordrecht: Springer.

Llopart Cudié, X., Campbell, M., Dinapoli, R., San Segundo, D. \& Pernigotti, E. (2002). IEEE Trans. Nucl. Sci. 49, 2279-2283.

McMullan, G., Cattermole, D. M., Chen, S., Henderson, R., Llopart, X., Summerfield, C., Tlustos, L. \& Faruqi, A. R. (2007). Ultramicroscopy, 107, 401-413.

McMullan, G., Chen, S., Henderson, R. \& Faruqi, A. R. (2009). Ultramicroscopy, 109, 1126-1143.

Plackett, R., Ballabriga, R., Campbell, M., Llopart, X., Tick, T., Tlustos, L., Turecek, D., Vahanen, S. \& Wong, W. (2010). PoS (VERTEX 2010), 030. http://pos.sissa.it/archive/conferences/113/ 030/VERTEX\%202010_030.pdf.

Rupp, B. (2004). Protein Crystallization Strategies for Structural Genomics, edited by N. Chayen, pp. 9-56. La Jolla: International University Line.

San Segundo Bello, D., van Beuzekom, M., Jansweijer, P., Verkooijen, H. \& Visschers, J. (2003). Nucl. Instrum. Methods Phys. Res. A, 509, 164-170.

Turecek, D., Holy, T., Jakubek, J., Pospisil, S. \& Vykydal, Z. (2011). J. Instrum. 6, C01046.

Visser, J., van der Heijden, B., Weijers, S. J. A., de Vries, R. \& Visschers, J. L. (2011). Nucl. Instrum. Methods Phys. Res. A, 633, S22-S25.

Vykydal, Z., Jakubek, J. \& Pospisil, S. (2006). Nucl. Instrum. Methods Phys. Res. A, 563, 112-115.

Zhang, X., Jin, L., Fang, Q., Hui, W. H. \& Zhou, Z. H. (2010). Cell, 141, 472-482. 